\title{
Gender And Stress: Does One's Gender Affect Stress Levels During An Evaluation?
}

Marian C. Schultz, (E-mail: mschultz@uwf.edu), The University of West Florida

James T. Schultz, (E-mail: james.schultz@erau.edu), Embry Riddle Aeronautical University

\begin{abstract}
This study sought to determine if there is a significant difference between male and female stress levels during evaluations. The research hypothesis states that there is a significant difference between the level of stress experienced between males and females during an evaluation. The ability to interpret a person's skin temperature has become a useful technique in biofeedback studies (Roberts, Kewman \& MacDonald, 1973). The instrument utilized in this study to measure the level of stress which individuals experience was the "Biodot", a one-quarter inch diagonal circle of microencapsulated Cholesteric liquid crystals which changes color to reflect a broad thermal range. The study, composed of 860 individuals, found that there was no significant difference between the stress levels of males and females during an evaluation.
\end{abstract}

\subsection{Male versus Female Stress}

T

he term stress has a variety of meanings. The Merriam-Webster dictionary defines stress as "to subject to hardship, affliction, or oppression" (1976, p. 2260). Stress, however, is more than its dictionary definition. Selye, in his book, Stress Without Distress, describes stress as "the nonspecific response of the body to any demand made upon it" (1974, p. 27). Selye explains that each demand made upon our body is nonspecific and unique. In other words, people react differently when faced with the same stressors. To fully understand Selye's definition, it is critical to comprehend what is meant by nonspecific. When exposed to cold, humans shiver to produce more heat, and the body vessels in our skin contract. Similarly, when exposed to heat, humans sweat because the evaporation of sweat from our skin has a cooling effect. Both of these demands have one thing in common; they increase the demand for readjustment. This demand is nonspecific, it requires our body to make an adjustment and it does not care which specific problem caused this adjustment to occur.

Stress may be the body's reaction to intense anger, or it may be the reaction to extreme success. Stress even occurs in animals and plants that function without a nervous system: it is not something that can be avoided. Individuals are constantly under some type of stress. When someone is stressed, it is perceived that he or she is under excessive stress or in distress. It is virtually impossible to be completely exempt from stress. While everyone experiences some sort of excessive stress from time to time, individuals all react differently to those stressors.

\subsection{The Body's Reaction To Stress}

Individuals react differently to stress. Some experience tension in their muscles, increased heartbeat, irritability, low back pain, insomnia, and fatigue are just a few examples. These signs are an indication that lifestyles need to be reevaluated, resolving a critical personal need to eliminate these conditions. Prolonged stress can affect one's job performance and lead to physical or emotional illness. Individuals, however, will react differently when confronted with the same stressful situation. According to Selye, the body copes with stress in three ways:

Readers with comments or questions are encouraged to contact the authors via email. 
- $\quad$ Stress messages travel from the brain through motor nerves to the action muscles in the arms, legs, and skeletal system, preparing for sudden explosive motion.

- $\quad$ The messages travel from the brain through the autonomic nerves to vital organs, increasing heart rate, blood pressure, blood sugar levels, respiration, and red blood cell count. This, in turn, increases the supply of oxygen and energy available to the body. The same pathway also slows down intestinal movement, since digestion yields to action in an emergency.

- $\quad$ Stress messages travel from the brain to the adrenal gland and the hypothalamus. The adrenal gland regulates the release of adrenalin into the blood stream as a fast-acting general stimulant. The hypothalamus, the emotion center of the brain, signals the pituitary and the adrenal cortex to release hormones into the bloodstream that offer slow-acting stress protection. The hormones alter the salt/water balance of the blood to raise blood pressure: stimulate the release of thyroid hormones to speed up metabolism, allowing rapid conversion of food to energy; and raise the white blood cell count, affecting some immune and allergic responses (Witkin-Lanoil, 1968 p. 27).

\subsection{Male versus Female Stress}

One way to explain the differences is to consider both male and female reactions to the stress stimuli. While males and females share much in common, they differ significantly when confronted with the same stressful situation. Women are more vulnerable to stress because of their life styles. Witkin-Lanoil, in her book The Male Stress Syndrome, calls this double jeopardy for women. "Women are in a double jeopardy for stress symptoms, because they have a reproductive system that both creates stress and is vulnerable to the effects of stress" (1986, $p$. 9). Witkin-Lanoil is indicating that women suffer the effects of menstrual disorders, migraine headaches, anxiety attacks, depression, and eating disorders such as anorexia. Many of the causes of male depression and stress arise from their self-nurtured identities, especially related to their professional status. When asked who they are, men are quick to respond with I'm a doctor, I'm a truck driver, and so forth. Men tend to let stress build and are at a higher health risk. Witkin-Lanoil explains "Men, however, find themselves at a higher risk of fatality from their stress symptoms. These include high blood pressure, high cholesterol, heart attacks, alcoholism and peptic ulcers" (1986, p. 9).

It has been noted that women tend to have a higher occurrence of stress and migraine headaches than men. Though a man's blood pressure rises more sharply in response to stress, women react to a wider range of stressors and say they feel stress more often, perhaps because they take a holistic view of everyday life. It was also found significant differences between men and women. Women's blood pressure goes up less than men's in reaction to stress (although their response increases noticeably after menopause or hysterectomy, suggesting a buffering effect from estrogen). Women tend to react to a wider range of outside stressors than men. The study asked 166 married couples to keep a daily diary for six weeks. It was determined that women feel stress more often because they take a holistic view of everyday life. A man may worry if someone in his immediate family is sick; his wife takes on the burdens of the whole neighborhood. "Men take care of one thing [at a time]", while "women put the pieces together again" (Kalb \& Rogers, 1999).

\subsection{Biofeedback}

Biofeedback is a process in which individuals voluntarily control bodily functions that are normally involuntary actions. The "biofeedback" term was developed in 1969 to describe laboratory-training techniques that have been in use since the 1940s. These techniques teach people to monitor the signals their body provides them in order to alter such things as brain activity, heart rate, muscle tension, blood pressure, skin temperature, etc. ("What is," 2002).

Biofeedback, although a relatively new scientifically-based medical procedure and was not widely recognized as being very beneficial, has recently emerged as a feasible treatment for some physical and emotional conditions. In a clinical session, patients are connected to sophisticated biofeedback machines, that measure single 
or multiple bodily functions, while being monitored and treated by a therapist. Through the therapy, the patient's awareness of his or her individual bodily functions is heightened. Once the patient is aware of how their body reacts to different stimuli, they can then self-regulate and control some "involuntary" bodily functions. Altering these body functions allows the patient to combat items like stress and headaches that are associated with many average daily activities (Anderson, 2000).

\subsection{Measurement Devices}

There are six common types of biofeedback measuring devices, and each device is used to target specific treatments. The three main types are Electromyographic (EMG), brainwave/neurofeedback (EEG), and thermal. Other types of biofeedback include Skin Conductance Level (SCL), Heart Rate (HR), and respiratory (Brauer, 1999).

The EMG device measures muscular tensions, while the more complicated EEG device measures brainwave activity. The SLC device measures sweat gland activity and the HR device measures rate and pulse volume. The respiratory device measures exhaled carbon dioxide and abdominal excursions. The most simplistic of all the devices, the thermal device, measures skin temperature (Brauer, 1999).

Interestingly, all of the aforementioned devices, except one, require expensive machinery and therapist office visits for treatments. The thermal device, due to its simplistic nature can be employed in an individual's home or office environment on a weekly, daily, or even hourly basis. The use of an inexpensive finger thermometer allows the individual to practice self-regulation at his or her own pact (Farmer, 1999).

These finger thermometers range in various sizes and types, and are easily available. Commonly called "biodots" or "biocards," the thermometers consist of a black strip on a plastic card or an adhesive pencil eraser-size dot that is held against the skin on the hand or finger. After a few minutes, the black indicator changes colors to reflect skin temperature.

\subsection{Skin Temperature}

The average finger temperature for a normal person is 85 degrees Fahrenheit. A lower finger temperature indicates higher stress levels. This change in temperature occurs as the natural instinct of the human body to redirect blood flow away from the extremities during times of high stress or fear. It is not uncommon for individuals with an extremely high stress level, sometimes resulting in headaches, to have a finger temperature in the 70s (Farmer, 1999). Similarly, when an individual is relaxed, their finger temperature increases. The goal for self-regulated therapy is to raise the finger temperature to 96 degrees Fahrenheit. Once the individual achieves this level of control over the cardiovascular system, bodily relaxation occurs, reducing the stress levels, and even eliminating headache pains (Farmer, 1999).

In order for an individual to gain control over their blood flow and increase the finger temperature, they must practice some type of self-nurturing activity. This activity could be anything from a massage to meditation. Such meditation activities could include 10-30 minutes of deep breathing, soothing music, and/or mind focusing. All geared toward voluntarily controlling and calming the body. The difficulty, however, is to find time in today's busy schedule for relaxing activities. The reward for the few that attain relaxation is being able to maintain a healthy state and a less stressed lifestyle (Farmer, 1999).

The ability to interpret a person's skin temperature has become a useful technique in biofeedback studies (Roberts, Kewman \& MacDonald, 1973). The reaction of microencapsulated Cholesteric liquid crystals (MCLC) to various skin temperatures can be used to readily identify the homeostatic (state of arousal) condition being experienced by an individual. One MCLC product, the Biodot, is temperature sensitive and changes color in accordance with the user's skin temperature, since skin temperature is purported to reflect a particular mood change in an individual (Schultz, Schultz \& Williams, 1986). In order to understand the theory associated with Biodot functions, it is important to understand the operants that regulate the skin's temperature. The autonomic nervous 
system (ANS) which controls the activity of our internal organs, glands, heart, lungs, and all the smooth muscles of the body, is made up of two opposing networks: the sympathetic nervous system (SNS) and the parasympathetic nervous system (PNS). The SNS is activated in times of stress. Under duress, the body automatically undergoes vasoconstriction causing an increase in the amount of blood flowing to vital organs. Simultaneously, blood moves away from the extremities causing the temperature in these areas to decrease (Gatchel \& Price, 1979), resulting in a color change in the Biodot (Schultz, 1986, 1987, 1988, 1990, 2002; Jenson, 1993). The PNS functions at slow metabolic rates or, at times, at rest. In this state, the blood flow is regulated throughout the body in a constant manner, causing the temperature of the skin to be higher than in times of stress (Gatchel \& Price, 1979).

Biodots, developed by Biodots International, Inc., contain a spectrum of color capabilities. Each color variance corresponds to a given skin temperature. The Biodot is a small flat device, approximately one-quarter inch in diameter, with a small amount of adhesive on the back enabling it to adhere to the skin without temperature interference. Biodots are effective biofeedback devices due to the small size, quick reaction time, and ease of interpretation. According to data provided by the manufacturer, the ideal location to place the Biodot is on the back of the hand, between the thumb and index finger. For individuals with poor blood circulation, the Biodot should be placed on the upper and innermost portion of the forearm in order to more accurately reflect changes in the skin's temperature. For accuracy and consistency in reading the Biodot, placement directly on the veins, arteries, or bones is not recommended (Biodot International, 2001).

Six previous studies utilized Biodots as a method of utilizing skin temperature to measure stress levels. Schultz, Schultz, and Williams (1986) investigated the differences in stress levels for business students taking written examinations and giving oral presentations. A second study by Schultz, Schultz, and Becker (1987) determined if a students' stress level, prior to an examination, could predict final test scores. A third study by Schultz, Leptrone, and Schultz (1988) measured stress levels created by various types of examinations. The fourth study by Jenson, Schultz and Schultz (1993) evaluated stress variations occurring during evaluations. The fifth study by Schultz, Schultz and Riley (1990), as well as the sixth study by Schultz and Schultz (2002), evaluated the affects on performance resulting in variations in stress levels.

According to Barrios, the inventor of the stress control card, it is extremely important for an individual to detect stressful situations in order to avoid subsequent stress-related problems, for example high blood pressure. As an alternative to the Biodot, the stress control card has successfully reduced many stress related problems because of its ability to detect temperature changes indicative of stress (Mullich, 1984).

\subsection{Statement Of Hypothesis}

The research hypothesis states that there is a significant difference in the level of stress experienced during an evaluation between males and females. The null hypothesis states that there is no significant difference in the level of stress experienced between males and females during an evaluation, as measured at the $\partial=.05$ level of significance. The significance of this research is to determine whether gender has an affect on stress levels during an evaluation. If it is determined that stress levels experienced under specific situations can minimize one's performance effectiveness, then preventative measures can be taken to reduce the level of stress.

\subsection{Research Methodology}

The survey population consisted of 860 students who were enrolled in classes with The University of West Florida at their Pensacola and Fort Walton Beach locations, and Embry-Riddle Aeronautical University at the Pensacola, Tampa, Fort Walton Beach, and Hurlburt AFB, Florida; and Charleston and Shaw AFB, South Carolina locations.

Prior to the study, participants were asked whether there were any external emotional circumstances that could have an affect on their stress level during the study. These external factors could include a death in the family, loss of job, unexpected pregnancy, etc. Participants who admitted to experiencing a highly stressful situation at anytime during the study were excluded. 
The survey population was $60 \%$ male (516) and $40 \%$ female (344). Ethnic origin was not deemed to be a factor since there is no conclusive research in this area that would indicate a significant difference between different ethnic groups. All three groups were approximately 60\% Caucasian, 20\% African-American, 15\% Hispanic and 5\% "other".

The instrument utilized to measure the level of stress which individuals experience was the "Biodot", a one-quarter inch diagonal circle of microencapsulated Cholesteric liquid crystals which changes color to reflect a broad thermal range. These items are manufactured by the Medical Device Corporation and are distributed by Biodot International, Inc. of Indianapolis, Indiana. According to the manufacturer, the colors of the Biodots reflect the following temperatures and physiological states (Table 1).

Table 1. Biodot Temperature and Physiological States

\begin{tabular}{|l|c|c|}
\hline Color & Temperature & Physiological State \\
\hline Black & 87 & Very Tense \\
\hline Amber & 89.6 & Tense \\
\hline Yellow & 90.6 & Unsettled \\
\hline Green & 91.6 & Involved \\
\hline Turquoise & 92.6 & Relaxed \\
\hline Blue & 93.6 & Calm \\
\hline Violet & 94.6 & Very Relaxed \\
\hline
\end{tabular}

The Biodot colors were assigned numerical values for the purpose of conducting the probability tests. The following colors were assigned the corresponding numerical values in Table 2 .

Table 2. Biodot Colors and Numerical Values.

\begin{tabular}{|c|c|}
\hline Color & Value \\
\hline Black & 1 \\
\hline Amber & 2 \\
\hline Yellow & 3 \\
\hline Green & 4 \\
\hline Turquoise & 5 \\
\hline Blue & 6 \\
\hline Violet & 7 \\
\hline
\end{tabular}

Prior to recording the numerical data, the Biodots were checked to ensure the colors reflected the temperature range indicated in Table 1. This was accomplished by using a heating element with predetermined temperature settings. Three thermostatic devices were utilized to check the temperature of the element. For further accuracy of the Biodot readings, the classroom temperatures were maintained between 70 degrees and 78 degrees. Also, the evaluations were scheduled during periods when there were no other major evaluations scheduled for the students.

\subsection{Procedures}

The researcher placed the Biodots on the back of the hand of each participant, between the thumb and index finger. Individuals, who admitted to a prior medical history of poor circulation, had the Biodots placed on the upper and innermost portion of the forearm. The placement of the Biodots was in accordance with medical information supplied by Biodot International (2001). Stress levels were recorded 20 minutes after the evaluations were distributed. To control for any variations which could occur with different individuals reading and recording the 
Biodot levels (colors) only the authors performed this particular task. The results of the two-dimensional Chi-Square test, found that a statistically significant relationship does not exist, at the .05 level of significance, between the level of stress experienced during an evaluation and the scores achieved.

Table 3. Biodot Colors

\begin{tabular}{|c|c|c|c|c|c|c|c|}
\hline & 7 & 6 & 5 & 4 & 3 & 2 & 1 \\
\hline Males & 79 & 86 & 96 & 89 & 96 & 41 & 29 \\
\hline Females & 64 & 61 & 44 & 71 & 54 & 24 & 26 \\
\hline \multicolumn{8}{|l|}{ Degrees of Freedom (df) 6} \\
\hline Level of Significance $\quad .05$ & & & & & & & \\
\hline Significance Value $\quad 12.59$ & & & & & & & \\
\hline Chi Square 9.515 & & & & & & & \\
\hline
\end{tabular}

\subsection{Conclusions}

The study found that there was no relationship in the level of stress experienced between males and females during an evaluation. These results reinforce the theory that the stress level of optimum performance will vary with each individual, regardless of gender. In some cases, individuals experiencing high levels of stress excelled in the appraisals while others did not. The same held true for individuals experiencing low degrees of stress. The research hypothesis for this study stated that there would be a significant difference between the stress levels of males and females during an evaluation. The data did not support the hypothesis. The null hypothesis for the study was not rejected since no significance existed between the genders. The researchers had perceived that there would be a difference in stress levels due to information gathered in prior studies which related to how males and females cope with stress in different situations.

\subsection{Recommendations}

While this study failed to show any significance difference between the stress levels between males and females during an evaluation, it did not consider the scores which individuals received on the evaluation. Past research has revealed that there is no relationship between the level of stress experienced by individual during an evaluation and the scores which they receive (Schultz \& Schultz, 2002). However neither past studies nor this research has taken into account the relationship of the three variables (gender, stress level, and evaluation score) to each other. Further research may find that a relationship between these three variables may exist. Other research might consider the effect various types of evaluations might have when considering gender and stress levels. This study only quantified data from one type of an evaluation. Further study, which could include the real time simulations and oral evaluations, may reveal significant differences between the genders.

\section{References}

1. Anderson, A. (2000). "Managing Stress with Biofeedback". Retrieved June 10, 2002 from http://www.athealth.com/ Consumer/farticles/Anderson.html

2. Biodot Interntional, Inc. (2001). Teacher's Guide.

3. Brauer, A. (1999) "Biofeedback and anxiety.” Retrieved June 10, 2002 from http://www.geocities.com/Wellesley/ Garden/8988/biofeedbackanxietyindex.htm

4. $\quad$ Farmer, K. U. (1999). "Biofeedback for stress and headaches.” The Pain Practitioner, 9 (4), pp. 1-2.

5. Gatchel, R.J., \& Price, K.P. (1979). "Biofeedback: an introduction and historical overview.” In R.J. Gatchel and K.P. Price (Eds.), Critical Applications of Biofeedback: Appraisal and Status. New York Pergamon Press.

6. Jenson, T., Schultz, J., \& Schultz, M. (1993). “Affects on performance resulting from stress variations occurring during evaluations." Paper presented at North American Economic and Finance Association $8^{\text {th }}$ International Congress, Montego Bay, Jamaica, August 1993. 
7. $\quad$ Kalb, C., \& Rogers, A. (1999). “Gender and Stress.” Newsweek.

8. $\quad$ Merriam-Webster's third new international dictionary (17 ${ }^{\text {th }}$ ed.). (1976). Philippines: G. \& C. Merriam Co.

9. Mullich, J. (1984). “A device for reducing stress?” Los Angeles Times, April 3, Part 5.

10. Roberts, A. H., Kewman, D.G., \& MacDonald, I. (1973). "Voluntary control of skin temperature: unilateral changes

11. using hypnosis and feedback.” Journal of Abnormal Psychology, Vol. 82, 63-168.

12. Schultz, M., Schultz, J. \& Williams, (1986). "Stress levels experienced by business students prior to oral presentations and written evaluations." Paper presented at the Eastern Region Conference of the Association of Human Resources Management and Organizational Behavior, New Orleans, Louisiana.

13. Schultz, M., Schultz, J., \& Becker, J. (1987). "A study of the relationship between examination scores and the degree of stress exhibited by individuals before, during and after examinations." Paper presented at the International Atlantic Economic conference, Munich, Germany.

14. Schultz, M., Leptrone, J., \& Schultz, J. (1988). "Utilizing Biodots to measure stress levels created by various types of examinations." Paper presented at the International Atlantic Economic Conference, London, England.

15. Schultz, M., Schultz, J., \& Reilly, T. (1990). "Affects on performance resulting from variations in stress levels occurring during evaluations." Journal of the Southwestern Society of Economists, Vol 17, No. 1, 73-77.

16. Schultz, J. \& Schultz, M. (2002). "The use of microencapsulated cholesteric material as a biofeedback mechanism to Measure the Relationship between Stress Levels and Performance." Published in the proceedings for the 2002 Hawaii International Conference on Business, Honolulu, Hawaii.

17. Selye, H. (1974). Stress without distress. Philadelphia \& New York: J.B. Lippincott Company.

18. What is biofeedback? (2002) Retrieved June 10, 2002 from the Association for Applied Psychophysiology and Biofeedback Website: http://www.aapb.org/public/articles/details.cfm?id=4

19. Witkin-Lanoil, G. (1986). The male stress syndrome. New York: Newmarket Press.

Notes 
Notes 